\title{
MODELING MIXED BOUNDARY PROBLEMS WITH THE COMPLEX VARIABLE BOUNDARY ELEMENT METHOD (CVBEM) USING MATLAB AND MATHEMATICA
}

\author{
ANTHONY N. JOHNSON, T.V. HROMADKA II, M.T. HUGHES \& S.B. HORTON \\ Department of Mathematical Sciences, United States Military Academy, USA.
}

\begin{abstract}
The complex variable boundary element method or CVBEM is a numerical technique that can provide solutions to potential value problems in two or more dimensions by the use of an approximation function that is derived from the Cauchy integral equation in complex analysis. Given the potential values (i.e. a Dirichlet problem) along the boundary, the typical problem is to use the potential function to solve the governing Laplace equation. In this approach, it is not necessary to know the streamline values on the boundary. The modeling approach can be extended to problems where the streamline function is needed because there are known streamline values along the problem boundary (i.e. a mixed boundary value problem). Two common problems that have such conditions are insulation on a boundary and fluid flow around a solid obstacle. In this paper, five advances in the CVBEM are made with respect to the modeling of the mixed boundary value problem; namely (1) the use of Mathematica and MATLAB in tandem to calculate and plot the flow net of a boundary value problem. (2) The magnitude of the size of the problem domain is extended. (3) The modeling results include direct computation and development of a flow net. (4) The graphical displays of the total flownet are developed simultaneously. And (5) the nodal point location as an additional degree of freedom in the CVBEM modeling approach is extended to mixed boundaries. A demonstration problem of fluid flow is included to illustrate the flownet development capability.

Keywords: approximate boundary, collocation, complex variable boundary element method (CVBEM), complex variables, mixed boundary conditions, Mathematica, MATLAB, MATLink
\end{abstract}

\section{INTRODUCTION}

Several new advances in the complex variable boundary element method 'CVBEM' are presented. (1) The CVBEM is applied with the use of computer programs Mathematica, MATLAB and Matlink. These three programs are integrated together to utilize the matrix-solving capability of MATLAB with the graphics capability of Mathematica, among other features. Mathematica and MATLAB are linked using procedure calls based upon Matlink's Application Programming Interface or API. The CVBEM is then extended to solving mixed boundary value problems. Previously, this software capability was focused upon solving Dirichlet problems. For the CVBEM, the mixed boundary value problem is handled by specifying values of the potential function on the problem boundary where Dirichlet-type boundary conditions are known. Then, the values of the complex stream function are specified on the boundary where flux-type or conjugate-type boundary conditions are known. This ability of handling both types of boundary conditions by direct specification of conjugate function values appears to be unique to the CVBEM. (2) The magnitude of the size of the problem domain, as far as the number of nodes used in the model, is extended to several hundreds or even in the thousands. Previously, the number of nodes reported in the literature has been approximately one hundred or less. By harnessing the power of these three computer programs, the size of the mixed boundary value problem that can be analyzed using the CVBEM has been greatly extended. (3) The CVBEM modeling results include direct computation of a flownet for the mixed boundary value problem. Other numerical modeling techniques use 
auxiliary computational schemes to estimate the conjugate function results or flux estimates using the estimates for the potential function derived in the model effort. The CVBEM develops both the potential and the conjugate stream function directly in tandem and simultaneously. (4) The graphical displays of the total flownet for both the potential and stream functions are developed simultaneously by Mathematica from the developed CVBEM approximation function. The flownet plots not only cover the problem domain and boundary but additionally cover the space exterior of the problem domain. This ability to develop the CVBEM approximation and then visualize the flownet both interior and exterior of the problem domain and boundary provides further insight into the model user in improving the CVBEM approximation fit to problem boundary conditions. This can be done by the addition of nodes (i.e. adding more CVBEM basis functions) or moving nodes to different locations on the problem boundary or to locations exterior of the problem boundary and domain. (5) The ability to include the nodal point locations as additional degrees of freedom in the CVBEM modeling approach is extended to mixed boundary value problems.

\section{COMPLEX VARIABLE BOUNDARY ELEMENT METHOD (CVBEM)}

The CVBEM is a numerical method, which solves partial differential equations of the Laplace and Poisson type. Since the model's conception in 1984 [1,2], the CVBEM has been examined in numerous publications including Hromadka and Lai [3], Hromadka and Whitley [4], and most recently Hromadka and Whitley [5]. The CVBEM was also extended to three or more dimensions through the use of linear combinations of the two-dimensional model [6]. It has been the subject of many research papers and engineering applications such as heat transfer, stress-strain, fluid dynamics and groundwater flow.

The CVBEM has an advantage over some other numerical methods such as the finite element method (FEM) or finite difference methods (FDM) because the CVBEM does not require modeling nodal points to be defined on the problem boundary or in the interior of the problem domain. Both the FEM and FDM techniques require nodal points to be defined on the problem boundary and within the problem domain. First, the CVBEM also solves the governing PDE, while FEM and FDM are only able to develop approximations. Secondly, the CVBEM provides an approximation function that is continuous throughout the problem domain where the other methods do not. Thirdly, the CVBEM approximation function exists outside the problem domain, whereas the FEM and FDM approximations do not. Furthermore, the CVBEM is more useful than analytical methods such as a Fourier series expansion because it is not limited to a specific domain.

Recent research by Johnson et al. [7] has demonstrated that relatively large instances (1000 or more nodes) of the Dirichlet problem modeled using the CVBEM can be quickly solved using MatLAB. With the Dirichlet problem, it is only necessary to know the potentials on the boundary because there are no flux boundary conditions. This paper extends the CVBEM to solve mixed boundary value problems. The paper also demonstrates the recent advancement of using CVBEM nodal points specified exterior of the problem domain (and not on the problem boundary) showing that nodal point positioning (both on the problem boundary and exterior of the problem domain) is an additional set of modeling degrees of freedom available for approximation optimization.

\section{MATHEMATICAL MODEL}

Application of the CVBEM begins with a simply connected domain in the two-dimensional complex plane, $\Omega$, which is bounded by a simple closed contour, $\Gamma$. A graphical depiction of this domain can be seen in Fig. 1. 


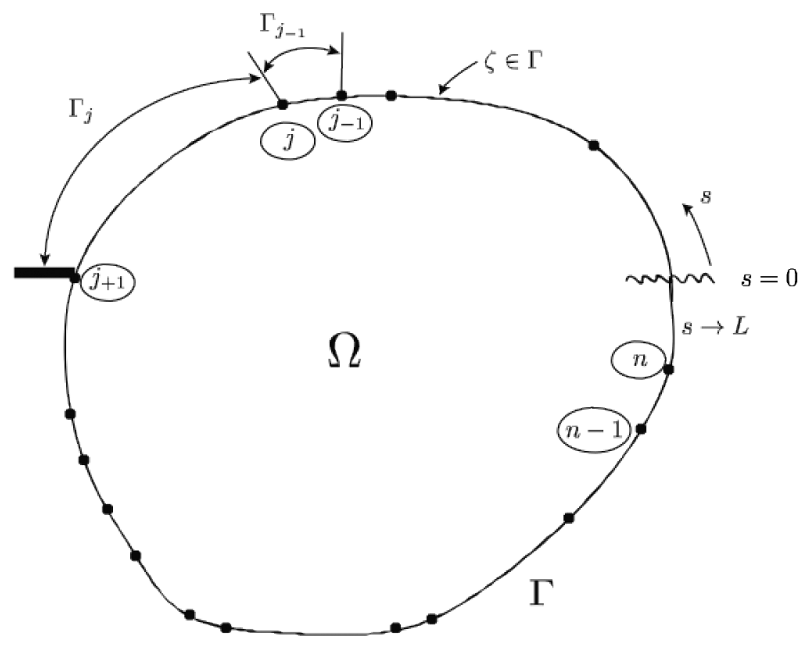

Figure 1: CVBEM [7].

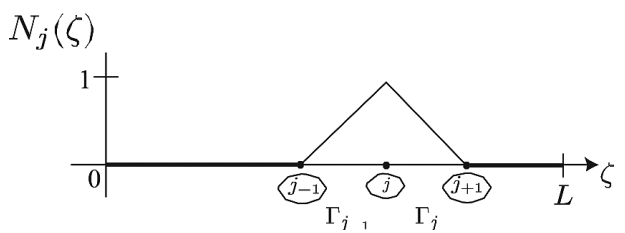

Figure 2: Linear interpolation basis functions, $N_{j}(\zeta)$.

Along the boundary $\Gamma$, or exterior to the problem domain union boundary, there are defined $n$ nodal points. For development purposes, the $n$ nodes are assumed defined on $\Gamma$. Next, the simple closed contour, $\Gamma$, is divided into $n$ boundary elements, $\Gamma_{j-1}, \ldots, \Gamma_{j}, \ldots, \Gamma_{n}$. For each boundary element, an interpolating polynomial will be used to create a piecewise continuous global interpolation function. In Fig. 1 , the boundary, $\Gamma$, is 'severed' at $s=0$ and in the positive direction spans until $s=L$, the arc length of $\Gamma$. In Fig. 2, the boundary is 'flattened' and the piecewise function is presented.

The piecewise function that is graphed in Fig. 2 is seen in the equation form,

$$
N_{j}(\zeta)= \begin{cases}\frac{\zeta-z_{j-1 \mid}}{z_{j}-z_{j-1}} & \zeta \in \Gamma_{j-1} \\ \frac{z_{j}+1-\zeta}{z_{j}+1-z_{j}} & \zeta \in \Gamma_{j} \\ 0 & \text { otherwise }\end{cases}
$$

Note that the sum of respective basis function terms is continuous on the boundary $\Gamma$ for all $\zeta \in \Gamma$. This basis function will be used to define the Global Trial function

$$
G_{n}(\zeta)=\sum_{j=1}^{n} N_{j}(\zeta) \varpi_{j}
$$


where the Global Trial function is defined to be the sum of all nodal basis functions multiplied by a corresponding complex coefficient, $\bar{w}_{j}$, where $\bar{w}_{j}$ is the nodal point $j$ value of the function being approximated.

The Global Trial function is substituted into the Cauchy integral for mula (eqn (3)). The integration of this formula with our analytic Global Trial function (in the simply connected two-dimensional complex domain, $\Omega$ ) results in the CVBEM approximation function, $\hat{w}\left(z_{0}\right)$ :

$$
\breve{\omega}\left(z_{0}\right)=\frac{1}{2 \pi i} \int_{\Gamma} \frac{G(\zeta) d \zeta}{\zeta-z_{0}}, \quad z_{0} \in \Omega .
$$

Because the Global Trial function is continuous, $\hat{w}(z 0)$ is analytic in $\Omega$, allowing $\hat{w}\left(z_{0}\right)$ to be used as an approximation function defined almost everywhere ('ae') inside $\Omega$ as well as exterior to $\Omega$ ae. This characteristic separates the CVBEM from other approximations such as FEM and FDM. When solved, the CVBEM approximating integral becomes an approximating function of the form

$$
\breve{\omega}(z)=P_{0}(z)+P_{1}(z)+\sum_{j=1}^{n}{ }_{j}\left(z-z_{j}\right) \ln _{j}\left(z-z_{j}\right)
$$

with

$$
C_{j}=\alpha_{j}+i \beta_{j}
$$

where $\alpha_{j}$ and $\beta_{j}$ are real constants to be determined, and

$$
P_{0}(z)=\left(\alpha_{0}+i \beta_{0}\right) \quad \text { and } \quad P_{1}(z)=\left(\alpha_{-1}+i \beta_{-1}\right)(x+i y)
$$

where $\alpha_{0}, \beta_{0}, \alpha_{-1}$ and $\beta_{-1}$ are also real constants to be determined. In eqn (4), the first terms are linear complex polynomials, which are added to a sum of products of linear complex polynomials multiplied by the nodal point complex logarithm. Since complex logarithms are used, it is necessary to expand on the definition of nodal point branch cuts.

A branch cut is a curve in the complex plane across which an analytic function is discontinuous. Since the approximation function $\hat{w}(z)$ can contain $n$ complex logarithmic terms corresponding to $n$ nodal points modeling the problem domain boundary, it is necessary to ensure that the discontinuities associated with each nodal point is rotated away from the domain under observation. Figure 3 shows the rotation of the branch cuts away from the problem domain, $\Omega$.

\section{CVBEM EXPANSION AND NODAL EQUATIONS}

The first step in applying the CVBEM is to resolve the CVBEM approximation function, $\hat{w}(z)$, into its real and imaginary parts, $\hat{\phi}(z)$ and $\hat{\psi}(z)$. The approximating function, eqn (4), is converted to polar coordinates. Then, the construct $\left(z-z_{j}\right)=R_{j} e^{i \theta j}$ is used to define the distance between any point $z$ and each node $j$, located at $z_{j}$,

$$
\breve{\omega}(z)=P_{0}(z)+P_{1}(z)+\sum_{j=1}^{n}\left(\alpha_{j}=i \beta_{j}\right) R_{j} e^{i \theta_{j}} \ln _{j}\left(R_{j} e^{i \theta_{j}}\right)
$$

Using Euler's formula of $e^{i \theta}=(\cos \theta+i \sin \theta)$, the CVBEM approximation function becomes 


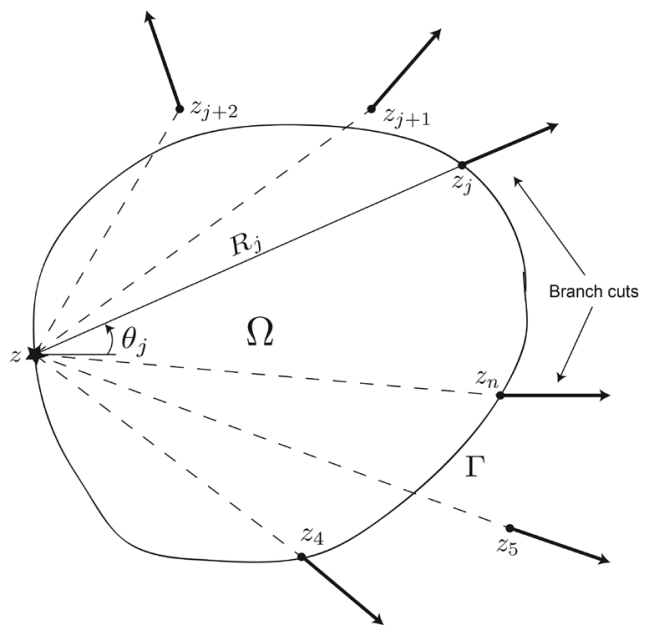

Figure 3: Branch cuts and polar coordinate development.

$$
\begin{aligned}
& \breve{\omega}(z)=\left(\alpha_{0}+i \beta_{0}\right)+\left(\alpha_{-1}+i \beta_{-1}\right)(x+i y)+ \\
& \sum_{j=1}^{n}\left(\alpha_{j}+i \beta_{j}\right) R_{j}\left(\cos \theta_{j}+i \sin \theta_{j}\right) \ln _{j}\left(R_{j} e^{i \theta_{j}}\right) .
\end{aligned}
$$

Further evaluation gives

$$
\ln _{j}\left(R_{j} e^{i \theta_{j}}\right)=\ln _{j}\left(R_{j}\right)+i \theta_{j}
$$

Combining eqns (6) and (7) gives

$$
\begin{aligned}
\left.\omega(z)=\alpha_{0}+i \beta_{0}\right)+\left(\alpha_{-1}+i \beta_{-1}\right)(x=i y)+\sum_{j=1}^{n}\left(\alpha_{j} R_{j} \cos \theta_{j}+\right. \\
\left.\quad i \alpha_{j} R_{j} \sin \theta_{j}+i \beta_{j} R_{j} \cos \theta_{j}+i^{2} \beta_{j} R_{j} \sin \theta_{j}\right)\left(\ln _{j}\left(R_{j}\right)+i \theta_{j}\right) .
\end{aligned}
$$

Collecting real and imaginary terms yields

$$
\begin{aligned}
& \breve{\omega}(z)=\left(\alpha_{0}+i \beta_{0}\right)+\left(\alpha_{-1}+i \beta_{-1}\right)(x+i y)+ \\
& \sum_{j=1}^{n}\left(\alpha_{j} R_{j}\left(\ln _{j}\left(R_{j}\right) \cos \theta_{j}-\theta_{j} \sin \theta_{j}\right)-\beta_{j} R_{j}\left(\ln _{j}\left(R_{j}\right) \sin \theta_{j}+\theta_{j} \cos \theta_{j}\right)+\right. \\
& \left.i\left[\alpha_{j} R_{j}\left(\ln _{j}\left(R_{j}\right) \sin \theta_{j}+\theta_{j} \cos \theta_{j}\right)+\beta_{j} R_{j}\left(\ln _{j}\left(R_{j}\right) \cos \theta_{j}-\theta_{j} \sin \theta_{j}\right)\right]\right) .
\end{aligned}
$$

Now the approximation function is separated into real, $\hat{\phi}$, and imaginary, $\hat{\psi}$, parts:

$$
\widehat{\omega}(z)=\hat{\phi}(z)+i \widehat{\psi}(z)
$$

where the potential functions, or real parts, are given by

$$
\hat{\phi}(z)=\alpha_{0}+\left(\alpha_{-1} x-\beta_{-1} y\right)+\Phi(z)
$$

for 


$$
\begin{aligned}
\Phi(z)=\sum_{j=1}^{n}\left(\alpha_{j} R_{j}\left(\ln _{j}\left(R_{j}\right) \cos \theta_{j}-\theta_{j} \sin \theta_{j}\right)-\right. \\
\left.\quad \beta_{j} R_{j}\left(\ln _{j}\left(R_{j}\right) R_{j} \sin \theta_{j}+\theta_{j} \cos \theta_{j}\right)\right)
\end{aligned}
$$

the stream functions, or imaginary parts, are given by

$$
\psi(z)=\beta_{0}+\left(\beta_{-1} x+\alpha_{-1} y\right)+\Psi(z)
$$

for

$$
\begin{aligned}
& \Psi(z)=\sum_{j=1}^{n}\left(\alpha_{j} R_{j}\left(\ln _{j}\left(R_{j}\right) \sin \theta_{j}+\theta_{j} \cos \theta_{j}\right)+\right. \\
& \left.\quad \beta_{j} R_{j}\left(\ln _{j}\left(R_{j}\right) \cos \theta_{j}-\theta_{j} \sin \theta_{j}\right)\right) .
\end{aligned}
$$

Recall that $\ln _{j}$ includes the effect of the nodal point logarithmic branch cut rotations.

\section{MATRIX FORMULATION}

After the real and imaginary parts of the CVBEM approximation equation have been developed, the next step is to find the constants, $\alpha_{n}$, and $\beta_{n}$, in the $\hat{\phi}$ and $\hat{\psi}$ functions. Currently, there are two methods commonly used to solve for the constants: collocation and least-squares minimization. This research uses the method of collocation to solve for the constants. In this method, the known potential and streamline values will be used to set up a system of equations to solve for the unknown constants. In past research, collocation was used to solve the real part of the CVBEM and required $2 n+3$ collocation points, where $n$ is the number of nodes. The need for $2 n+3$ collocation points arises from eqns (11) and (13). For every node, there is an $\alpha_{j}$ and $\beta_{j}$ term in the summation and two additional $\alpha$ terms and $\beta$ terms, but one $\beta$ term is zero due to eqns (11) and (13).

Example 5.1. A single node CVBEM model for singleton node $k$.

Consider the real portion of the CVBEM with one node for collocation point $k$. The resulting equation from eqn (11) is:

$$
\hat{\phi}_{k}(z)=\hat{\phi}_{k}\left(x_{k}+i y_{k}\right)=\alpha_{0}+\alpha_{1} x_{k}-\beta_{1} y_{k}+\alpha_{2} p_{1, k}-\beta_{2} q_{1, k}
$$

where

$$
\begin{aligned}
p_{n, k} & =R_{n, k}\left(\ln \left(R_{n, k}\right) \cos \theta_{n, k}-\theta_{n, k} \sin \theta_{n, k}\right. \\
q_{n, k} & =R_{n, k}\left(\ln \left(R_{n, k}\right) \sin \theta_{n, k}-\theta_{n, k} \cos \theta_{n, k}\right.
\end{aligned}
$$

Evaluate the above with the five necessary potential collocation points on the problem boundary. This will result in five linearly independent equations for five collocation points on $\Gamma$,

$$
\begin{aligned}
& \hat{\phi}_{k}(z)=\hat{\phi}_{k}\left(x_{1}+i y_{1}\right)=\alpha_{0}+\alpha_{1} x_{1}-\beta_{1} y_{1}+\alpha_{2} p_{1,1}-\beta_{2} q_{1,1} \\
& \hat{\phi}_{k}(z)=\hat{\phi}_{k}\left(x_{2}+i y_{2}\right)=\alpha_{0}+\alpha_{1} x_{2}-\beta_{1} y_{2}+\alpha_{2} p_{1,2}-\beta_{2} q_{1,2} \\
& \hat{\phi}_{k}(z)=\hat{\phi}_{k}\left(x_{3}+i y_{3}\right)=\alpha_{0}+\alpha_{1} x_{3}-\beta_{1} y_{3}+\alpha_{2} p_{1,3}-\beta_{2} q_{1,3} . \\
& \hat{\phi}_{k}(z)=\hat{\phi}_{k}\left(x_{4}+i y_{4}\right)=\alpha_{0}+\alpha_{1} x_{4}-\beta_{1} y_{4}+\alpha_{2} p_{1,4}-\beta_{2} q_{1,4} \\
& \hat{\phi}_{k}(z)=\hat{\phi}_{k}\left(x_{5}+i y_{5}\right)=\alpha_{0}+\alpha_{1} x_{5}-\beta_{1} y_{5}+\alpha_{2} p_{1,5}-\beta_{2} q_{1,5}
\end{aligned}
$$


The matrix system to be solved is $A x=b$, where $A$ is a coefficient matrix and $b$ are the known potential values at each of the collocation points:

$$
\left[\begin{array}{l}
\hat{\phi}_{1} \\
\hat{\phi}_{2} \\
\hat{\phi}_{3} \\
\hat{\phi}_{4} \\
\hat{\phi}_{5}
\end{array}\right]=\left[\begin{array}{lllll}
1 & x_{1} & -y_{1} & p_{1,1} & -q_{1,1} \\
1 & x_{2} & -y_{2} & p_{1,2} & -q_{1,2} \\
1 & x_{3} & -y_{3} & p_{1,3} & -q_{1,3} \\
1 & x_{4} & -y_{4} & p_{1,4} & -q_{1,4} \\
1 & x_{5} & -y_{5} & p_{1,5} & -q_{1,5}
\end{array}\right]\left[\begin{array}{l}
\alpha_{0} \\
\alpha_{1} \\
\beta_{1} \\
\alpha_{2} \\
\beta_{2}
\end{array}\right] .
$$

Once the system $\mathrm{h}$ as been configured, substitute the coordinates of the collocation points into the second and third column of the coefficient matrix. It is also necessary to calculate the radius and angle of the collocation points from the singleton node (see Fig. 4). The final step is to solve the matrix system. Once these values are known, they can be substituted back into the original equation for $\hat{\phi}(z)$, eqn (15). The $\hat{\phi}$ function can now be used to approximate all the potential values within the problem domain.

Collocation can also be used in the same way to solve for the streamline equation, $\hat{\psi}(z)$. The most significant change is that instead of solving for $\alpha_{0}$, one must solve for $\beta_{0}$.

\section{CVBEM MODELING OF MIXED BOUNDARY VALUE PROBLEM USING COMPUTER SOFTWARE: IDEAL FLUID FLOW MAKING A $90^{\circ}$ BEND}

In order to demonstrate the advances presented in this paper, a simple ideal fluid flow problem of flow in a $90^{\circ}$ bend is considered (that has an exact solution available for comparison purposes). Although much more complex mixed boundary value problems may obviously be handled with the presented approach, only a demonstration problem is presented.

An application of the CVBEM that uses computer programs MatLAB, Mathematica and MATLink to model mixed boundary conditions of an ideal fluid flowing around a $90^{\circ}$ bend is presented. The geometry of this problem begins with a unit square anchored at the origin. The sides extending from the origin to $(1,0)$ and $(0,1)$ are defined by the streamline condition, and the value along these two sides is defined as reference value zero. The constant value of zero means that there is no flux, or fluid flowing, across the boundary. By the definition of PDE mixed boundary conditions, derivatives normal to the function $w(z)$ would be zero.

The sides from $(1,0)$ to $(1,1)$ and $(1,1)$ to $(0,1)$ are defined by the potential boundary conditions. On these two sides, the value at each point is set equal to the equation $x^{2}-y^{2}$. For

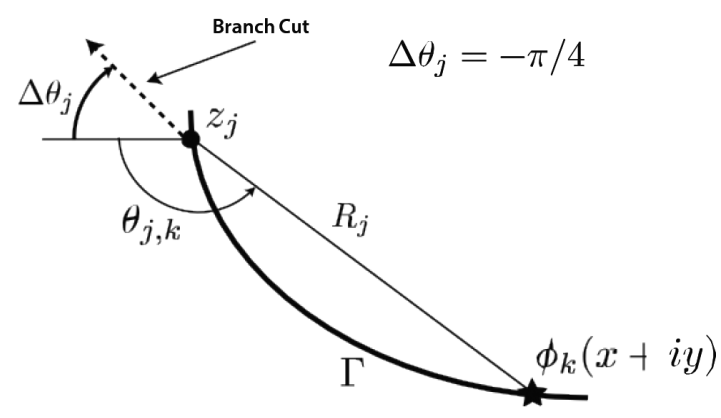

Figure 4: Solving for $R$ and $\theta$ in eqn (16). 


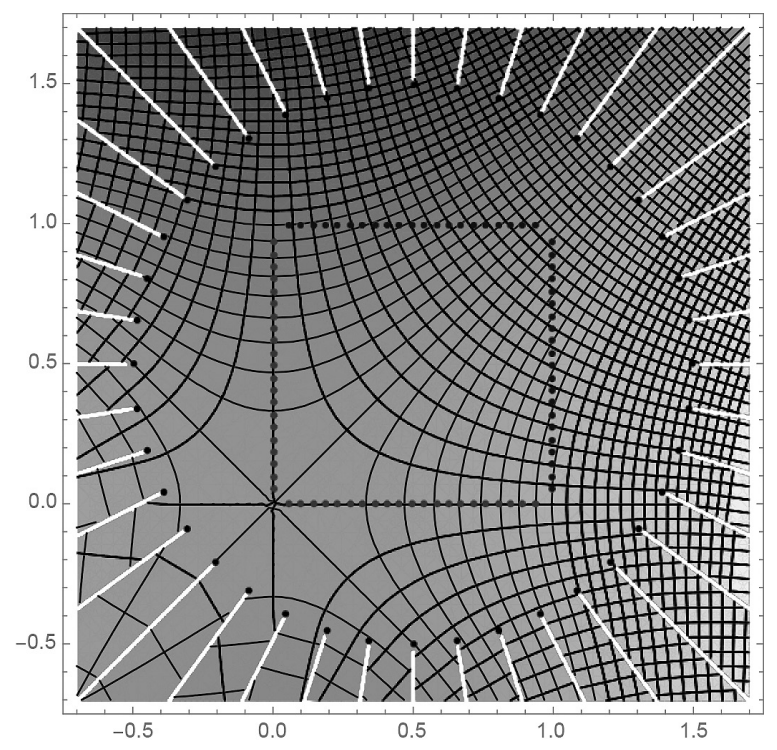

Figure 5: Ideal fluid flow around a $90^{\circ}$ turn.

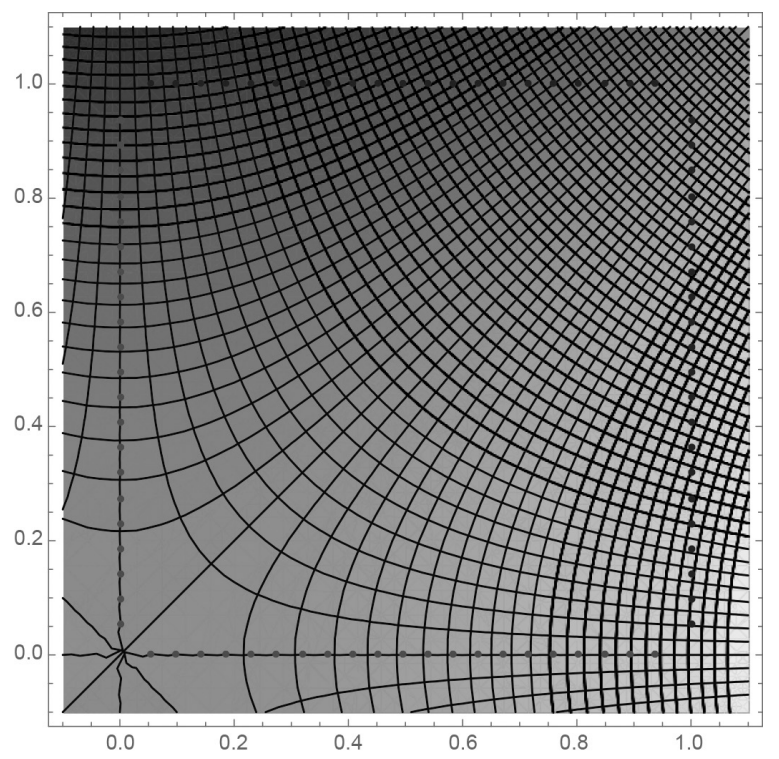

Figure 6: Zoom of boundary of ideal fluid flow around a $90^{\circ}$ turn.

example, the potential value at the evaluation point $(1,0.5)$ is equal to the value of 1.25 . The approximation function, $w(z)$, can be solved for the selected number of nodes and rotations of the various nodal branch cuts. In this problem, the number of nodes has been defined to equal forty. These forty nodes were placed in a circle around the center of the domain with a radius of $r=1$. The branch cuts are rotated $180^{\circ}$ from the angle of the node from the point $(0.5,0.5)$. This rotation will ensure that none of the branch cuts will cut through the problem domain. For the 40 nodes in this problem, there will be a corresponding $2 n+4$ collocation 
points, or 84 . Further, there are 42 potential value points, 21 on the right and 21 on the top of the problem boundary. Also, there are 42 streamline value points, 21 on the left and 21 on the bottom of the problem boundary. Because there are 40 nodes and 84 collocation points, there will be a system of 84 equations solving for 84 coefficients. MATLAB was able to handle this calculation easily. A Mathematica graphical contour plot of streamlines and potentials is shown in Fig. 5. Figure 6 is a zoom of the boundary only. Nodal points are outside the zoom window.

\section{CONCLUSIONS}

The CVBEM is a powerful numeric technique useful in solving the Laplace and Poisson equations. In this paper, several new advances in the CVBEM were demonstrated. With Matlink, the matrix-solving capability of MATLAB and the graphics capability of Mathematica were used to extend the number of nodes used to model the CVBEM mixed boundary value problem. Previously, this software capability was focused upon solving Dirichlet problems. The ability to handling both types of boundary conditions by direct specification of conjugate function values was demonstrated. Further the magnitude of the size of the problem domain, as far as the number of nodes used in the model is scalable. Previously, the number of nodes reported in the literature has been approximately one hundred or less. This implementation can handle thousands of nodes. Consequently, the size of the mixed boundary value problem that can be analyzed has been greatly extended. The graphical displays of the total flownet for both the potential and stream functions were developed simultaneously by Mathematica from the developed CVBEM approximation function as seen in both the examples. The flownet plots not only cover the problem domain and boundary but additionally cover the space exterior of the problem domain. This analysis has demonstrated the ability to develop the CVBEM approximation and then visualize the flownet both interior and exterior of the problem domain and boundary, and provides further insight into the modeler for deeper analysis. Finally, the nodal point locations were exploited as additional degrees of freedom in the CVBEM modeling of the mixed boundary value problems. Consequently, the approximation effort has not only the nodal point coefficients (of the CVBEM basis functions) as degrees of freedom in the approximation optimization, but also nodal point locations both on the problem boundary and also exterior of the problem domain and boundary. This extension of the CVBEM by including node location as a degree of freedom substantially increases the CVBEM modeling power in developing optimized approximations to mixed boundary value problems.

\section{REFERENCES}

[1] Hromadka, T.V. \& Guymon, G.L., The complex variable boundary element method. International Journal for Numerical Methods in Engineering, 1984.

[2] Whitley, R.J. \& Hromadka, T.V., Theoretical developments in the complex variable boundary element method. Engineering Analysis with Boundary Elements, 30(12), pp. 1020-1024, 2006. doi: http://dx.doi.org/10.1016/j.enganabound.2006.08.002

[3] Hromadka, T.V. \& Lai, C. The Complex Variable Boundary Element Method, SpringerVerlag: New York, NY, 1987.

[4] Hromadka, T.V. \& Whitley, R.J., Advances in the Complex Variable Boundary Element Method, Springer: New York, NY, 1998.

[5] Hromadka, T.V. \& Whitley, R., Foundations of the Complex Variable Boundary Element Method, Springer, 2014. 
[6] Hromadka, T.V. A Multi-dimensional Complex Variable Boundary Element Method. Topics in Engineering, Vol. 40. WIT Press: Billerica, MA, 2002.

[7] Johnson, A.N., Hromadka, T.V., Carroll, M., Hughes, M., Jones, L., Pappas, N., Thomasy, C., Horton, S., Whitley, R. \& Johnson, M., A computational approach to determining $\{\mathrm{CVBEM}\}$ approximate boundaries. Engineering Analysis with Boundary Elements, 41(0), pp. 83-89, 2014. doi: http://dx.doi.org/10.1016/j.enganabound.2013.12.011 